\title{
The prognostic effect of HER2 heterogeneity and YAP1 expression in HER2 positive breast cancer patients: a retrospective study
}

\author{
Jia-Qi Yuan, Zhi Xiao, Shou-Man Wang, Lei Guo^ ${ }^{\wedge}$ \\ Clinical Research Center for Breast Cancer Control and Prevention in Hunan Province, Multidisciplinary Breast Cancer Center, Department of \\ General Surgery, Xiangya Hospital, Central South University, Changsha, China \\ Contributions: (I) Conception and design: All authors; (II) Administrative support: SM Wang; (III) Provision of study materials or patients: JQ Yuan, L \\ Guo; (IV) Collection and assembly of data: JQ Yuan, Z Xiao, L Guo; (V) Data analysis and interpretation: JQ Yuan, L Guo; (VI) Manuscript writing: \\ All authors; (VII) Final approval of manuscript: All authors. \\ Correspondence to: Lei Guo. Clinical Research Center for Breast Cancer Control and Prevention in Hunan Province, Multidisciplinary Breast Cancer \\ Center, Department of General Surgery, Xiangya Hospital, Central South University, No. 87, Xiangya Road, Kaifu District, Changsha 410008, \\ China. Email: kehanshiming@163.com.
}

Background: This study sought to estimate the prognostic effect of intratumoral heterogeneity (ITH) and Yes-associated protein 1 (YAP1) intensity in human epidermal growth factor receptor 2 (HER2) positive breast cancer patients. We also investigated individualized adjuvant therapy for YAP1-sufficient patients and HER2 heterogeneous patients.

Methods: The relationship between prognostic outcomes and clinicopathological variables in 1,650 retrieved breast cancer patients was evaluated. The HER2 intensity and YAP1 expression in HER2-ITH and non-ITH (NITH) patients were also estimated. All patients were followed-up, regardless of whether or not they received intensive treatment, to explore individualized adjuvant therapy for YAP1-sufficient patients and HER2 heterogeneous patients.

Results: Over-expression and nuclear localization of YAP1 were significant in HER2-ITH patients. The over-expression of YAP1 and the presence of ITH affected the prognosis of HER2 positive patients. YAP1 intensity and lymph nodes metastases was more obviously affected the survival of HER2-ITH patients, while the prognosis of NITH patients were correlated with clinical Tumor-Node-Metastasis (cTNM) stage and lymph-vascular space invasion (LVSI) status. HER2-NITH patients and YAP1-insufficient patients benefited from a combination of Trastuzumab and Pertuzumab/Lapatinib, while Capecitabine significantly decreased the relapse risk of ITH patients and YAP1-sufficient patients.

Conclusions: YAP1 overexpression and nuclear localization was usually observed in HER2-ITH patients. For HER2-NITH patients, an advanced stage of cTNM and LVSI status increased the recurrent risk, and intensified Pertuzumab or Lapatinib treatment (combination with Trastuzumab) improved their survival. For HER2-ITH patients, the overexpression of YAP1 and pathological lymph nodes (pLN) metastases increased recurrent risk, and intensified Capecitabine treatment improved their survival. YAP1 overexpression contributed to a poor prognostic outcome, especially when HER2 signal intensity was insufficient.

Keywords: Breast cancer; human epidermal growth factor receptor 2 (HER2); Yes-associated protein 1 (YAP1); Trastuzumab; outcome

Submitted Dec 22, 2021. Accepted for publication Feb 14, 2022.

doi: $10.21037 / g s-22-52$

View this article at: https://dx.doi.org/10.21037/gs-22-52

^ ORCID: 0000-0002-3376-765X. 


\section{Introduction}

Nearly $25 \%$ of breast cancer cases present amplification of human epidermal growth factor receptor 2 (HER2) gene, which result in impaired disease-free survival and decreased overall survival (1). The routine use of Trastuzumab alters the natural course of HER2 positive breast cancer (1). Trastuzumab is a monoclonal antibody that targets the extracellular domain of HER2 protein and interferes with the HER2-mediated signaling cascade, preventing proliferation, and eventually leading to cell death (2). The perioperative use of Trastuzumab has been reported to improve pathological complete response rates and reduce relapse in HER2 positive patients $(3,4)$.

However, in our clinical practice, we have observed that some so-called "HER2-positive" patients have not benefited from anti-HER treatment. Pectasides et al. found that patients without HER2 amplification in recurrent lesions had shorter disease-free survival than HER2 positive relapse (5). The above findings prompted us to focus on the potential prognostic effect of HER2 intratumoral heterogeneity (HER2-ITH). According to the American Society of Clinical Oncology and College of American Pathologists (ASCO/CAP) 2013 guidelines, tumor cells with a HER2/centromere enumeration probe for chromosome (CEP17) signal ratio $>2.0$ and/or $>6$ HER2 signals per cell were considered to be HER 2 gene amplification. Thus, genetic HER2-ITH was defined as proportion of HER2 amplification cells ranged from $5 \%$ to $50 \%$ of the total cells (6). It may be that associations between risk factors and prognostic effects do not follow a linear pattern (7). Since the use of standard approaches is limited, we improved the analytic techniques used to detect these potentially complex associations.

The hippo pathway plays a crucial role in the therapeutic efficacy of Trastuzumab $(8,9)$, the central role of which is the degrading of Yes-associated protein 1 (YAP1) (10). According to the previous literatures, over-expression of YAP1 was significantly associated with the migration and invasion of tumor cells via inducing the inhibition of apoptosis, which contributed to tumorigenic and development of breast cancer $(11,12)$. A preliminary clinical study also revealed a statistically significant relationship between YAP1 and HER2 intensity in breast cancer patients (8).

In this study, we examined the occurrence ITH or nonITH (NITH) in HER2 positive patients. We explored the prognostic effect of HER2-ITH and its interaction with other variables, and evaluated the prognostic outcome of patients with or without HER2-ITH using the subpopulation treatment effect pattern plot (STEPP) methodology. We also investigated YAP1 expression in HER2-ITH and NITH patients to explore the relationship between HER2 heterogeneity and YAP1 intensity, and revealed that expression of YAP1 had a preferable relation with HER2 intensity in breast cancer tissues. Interestingly, over-expression of YAP1 was significant in HER2 heterogeneous breast cancer cases, which impaired the survival profoundly. We followed-up all patients who did or did not undergo intensified treatment, and sought to determine individualized strategies according to HER2-ITH and YAP1 expression. We present the following article in accordance with the MDAR checklist (available at https:// gs.amegroups.com/article/view/10.21037/gs-22-52/rc).

\section{Methods}

\section{Study population}

The data of 1,650 hormone receptor (HR)-/HER2+ breast cancer patients (clinical stages I-III) were retrieved from the database of the Breast Cancer Center, Xiangya Hospital Central, South University. All the enrolled patients underwent pathological diagnosis between January 2011 and January 2019. Patients who suffered from inflammatory breast cancer, or bilateral breast cancer, or distant metastasis disease were excluded from the study. All the enrolled patients were female, and had a median age of 47.5 years (range, 21-77 years). The median length of the followup period was 67 months (range, 13-108 months), and the follow-up period ended in September 2020. The study was conducted in accordance with the Declaration of Helsinki (as revised in 2013). The study was approved by the Xiangya Hospital Ethics Committee (approval No. 202004189) and individual consent for this retrospective analysis was waived.

The log-rank method was used to compare the difference in survival time between subgroups of patients. The mean time for breast cancer-free survival was estimated to be 48.5 months in the YAP1-insufficient group and 33.5 months in the YAP1-sufficient group [corresponding hazards ratio $(\mathrm{HR})=0.66]$, and each group needed to comprise 770 patients to achieve a $90 \%$ confidence level to detect the difference at a significant level of $\alpha=0.05$ (bilateral). It was planned that the trial would run for 
96 months with a censoring proportion less than 5\% (loss of follow-up, withdrawl from study, or abscission due to other diseases).

\section{Study design and procedures}

In this retrospective study, the diagnostic and therapeutic data were collected from the medical record system of Xiangya Hospital. In this study, all the patients enrolled received anthracycline and taxanes based chemotherapy (intravenously on day 1 , every 21 days for 8 cycles) and Trastuzumab adjuvant therapy $(6 \mathrm{mg} / \mathrm{kg}$, after a loading dose of $8 \mathrm{mg} / \mathrm{kg}$, every 3 weeks for 1 year). Radical surgery, such as breast-conserving surgery and modified radical mastectomy, was performed within 1 month of neoadjuvant therapy (NAT). The local advanced patients and breastconserving patients underwent normative radiation therapy according to NCCN clinical practice guidelines in breast cancer (version 2.2021). A total of 135 patients underwent intensified Pertuzumab treatment $(840 \mathrm{mg}$ for the first time and then $420 \mathrm{mg}$ every 3 weeks) in combination with the above-mentioned Trastuzumab treatment, 288 patients underwent intensified Lapatinib treatment $(1,250 \mathrm{mg} / \mathrm{d})$ in combination with the above-mentioned Trastuzumab treatment, and 387 patients underwent intensified Capecitabine treatment $\left(2,500 \mathrm{mg} / \mathrm{m}^{2} / \mathrm{d}\right.$ for 2 weeks and intermittent for 1 week). The above-mentioned intensified regimens were performed according to accessibility of targeted agents (e.g., Pertuzumab and Lapatinib) in a certain period.

The specimens were fixed with $10 \%$ neutral phosphatebuffered formalin immediately upon excision, and stained with hematoxylin and eosin (H\&E). The pathological results, such as HER2, Ki-67 indication, lymph-vascular space invasion (LVSI), and histological grade (histo-grade), were available for all patients. HER2 positive was defined as immunohistochemistry (IHC) staining HER2 $3+$ or fluorescence in situ hybridization (FISH)+. FISH positive was defined as a single-probe average HER2 copy number $\geq 6.0$ signals/cell, or a dual-probe HER2/CEP17 ratio of $\geq 2.0$ with an average HER2 copy number $\geq 4.0$ signals/cell, or a dual-probe HER2/CEP17 ratio of $<2.0$ with an average HER2 copy number $\geq 6.0$ signals/cell (6). According to the ASCO/CAP 2013 guidelines, HER2 genetic heterogeneity was defined as $5 \%$ to $50 \%$ of the total cells, with a HER $2 /$ CEP17 signal ratio $>2.0$, or $>6$ HER2 signals per cell (10). Anti-YAP1 was used to evaluate YAP1 status in HER2-ITH and NITH lesions. The expression levels of YAP1 were compared between paired tumor and para-tumor tissues. YAP1 expression was assessed by IHC staining and Western blot analysis (YAP1 antibody, Cat\# MA5-17200, RRID: AB_2538671), for which a positive result was confirmed when $>10 \%$ of the tumor cells were nuclear and/or showed cytoplasmic staining. YAP1 nuclear localization was estimated by Immunofluorescence analysis.

\section{STEPP basics and extensions}

The STEPP (13) methodology was used to examine interactions between the subgroups and the covariates by estimating the prognostic effect within overlapping subpopulations of patients. HRs were used in the STEPP analysis to describe the subgroup effects (14), and a competing risk analysis was conducted to estimate the interactive effects on the disease-specific endpoints (15). Associations among the risk factors and connections between treatment and survival were both explored by evaluating disease-specific events in the competing risk setting (16). The breast cancer-free percentage was determined to evaluate the prognostic outcome. Recurrence-free cases and survival cases were censored by the last follow-up time $(17,18)$. A Cox proportionalhazards model was constructed to estimate the composite measure of relapse risk. The continuous measure of relapse risk was determined by YAP1 intensity, clinical TumorNode-Metastasis (cTNM) stage, histo-grade, nodal status, LVSI status, and Ki-67 levels. For each enrolled patient, the value of composite risk was calculated by summing the model parameter estimates corresponding to the observed variable values. In this study, the STEPP methodology was used to investigate patterns in the absolute prognostic effect, as measured by Kaplan-Meier estimates of the breast cancer-free percentage (y-axis), across the continuum of the values of composite risk ( $\mathrm{x}$-axis). A nomogram analysis was also performed to estimate recurrent risk and survival probability.

\section{Statistical analysis}

A univariate analysis and the Pearson $\chi^{2}$ test were used to describe the baseline characteristics. The Cox proportionalhazards model was used to identify predictive factors of prognosis, and the results are expressed as HRs and 95\% confidence intervals (CIs). We compared the prognosis of subpopulations via a Kaplan-Meier survival analysis. The STEPP methodology was also used to evaluate the 
Table 1 Comparison of characteristics between HER2-ITH and non-HER2-ITH subpopulations

\begin{tabular}{|c|c|c|c|c|c|c|c|}
\hline Variable & \multicolumn{2}{|c|}{ No. of patients (\%) } & $\chi^{2}$ & $P\left(\chi^{2}\right)$ & OR & $95 \% \mathrm{Cl}$ & $P$ \\
\hline Age (years) & & & 0.299 & 0.585 & 1.135 & $0.826-1.477$ & 0.251 \\
\hline$\leq 47.5$ & $179(47.1)$ & $578(45.5)$ & & & & & \\
\hline$>47.5$ & 201 (52.9) & $692(54.5)$ & & & & & \\
\hline$\leq 50$ & $190(50.0)$ & $608(47.9)$ & & & & & \\
\hline$>50$ & $190(50.0)$ & $662(52.1)$ & & & & & \\
\hline Histological grade & & & 2.388 & 0.122 & 1.292 & $0.873-1.639$ & 0.126 \\
\hline I & $137(36.1)$ & $404(31.8)$ & & & & & \\
\hline Positive & $160(42.1)$ & $519(40.9)$ & & & & & \\
\hline Negative & $220(57.9)$ & $751(59.1)$ & & & & & \\
\hline Ki-67 (\%) & & & 0.977 & 0.323 & 1.234 & $0.851-2.030$ & 0.479 \\
\hline$>30$ & $111(29.2)$ & $405(31.9)$ & & & & & \\
\hline$\leq 30$ & $269(70.8)$ & $865(68.1)$ & & & & & \\
\hline pLN status & & & 0.556 & 0.456 & 1.522 & $0.704-2.098$ & 0.241 \\
\hline Positive & $165(43.4)$ & $579(45.6)$ & & & & & \\
\hline Negative & $215(56.6)$ & $691(54.4)$ & & & & & \\
\hline BCS & $156(41.1)$ & $504(39.7)$ & & & & & \\
\hline
\end{tabular}

*, measured via ultrasound and/or magnetic resonance imaging; HER2, human epidermal growth factor receptor; ITH, intratumoral heterogeneity; NITH, non-intratumoral heterogeneity; LVSI, lymph-vascular space invasion; axillary lymph nodes metastasis; YAP1, Yesassociated protein 1; BCS, breast-conserving surgery.

disease-specific cumulative incidence and composite recurrence risk. All the statistical tests were 2 -sides, and $\mathrm{P}$ values $<0.05$ were considered statistically significant. The STEPP and nomogram analyses were carried out with $\mathrm{R}$ software package version 4.0.4 (R Foundation for Statistical Computing, Vienna, Austria; https://sites.google.com/ site/stepprpackage, RRID: SCR_001905). SPSS version 19.0 for Windows (SPSS Inc., Chicago, IL, USA, RRID: SCR_002865) was used for the other statistical analyses.
Graphpad Prism version 8.0 for Windows (GraphPad Software Inc., San Diego, CA, USA, RRID: SCR_002798) was used to create figures.

\section{Results}

We compared the characteristics of the HER2-ITH and HER2-NITH subpopulations. As Table 1 shows, HER2ITH patients tended to have a positive YAP1 status $(69.7 \%$ 

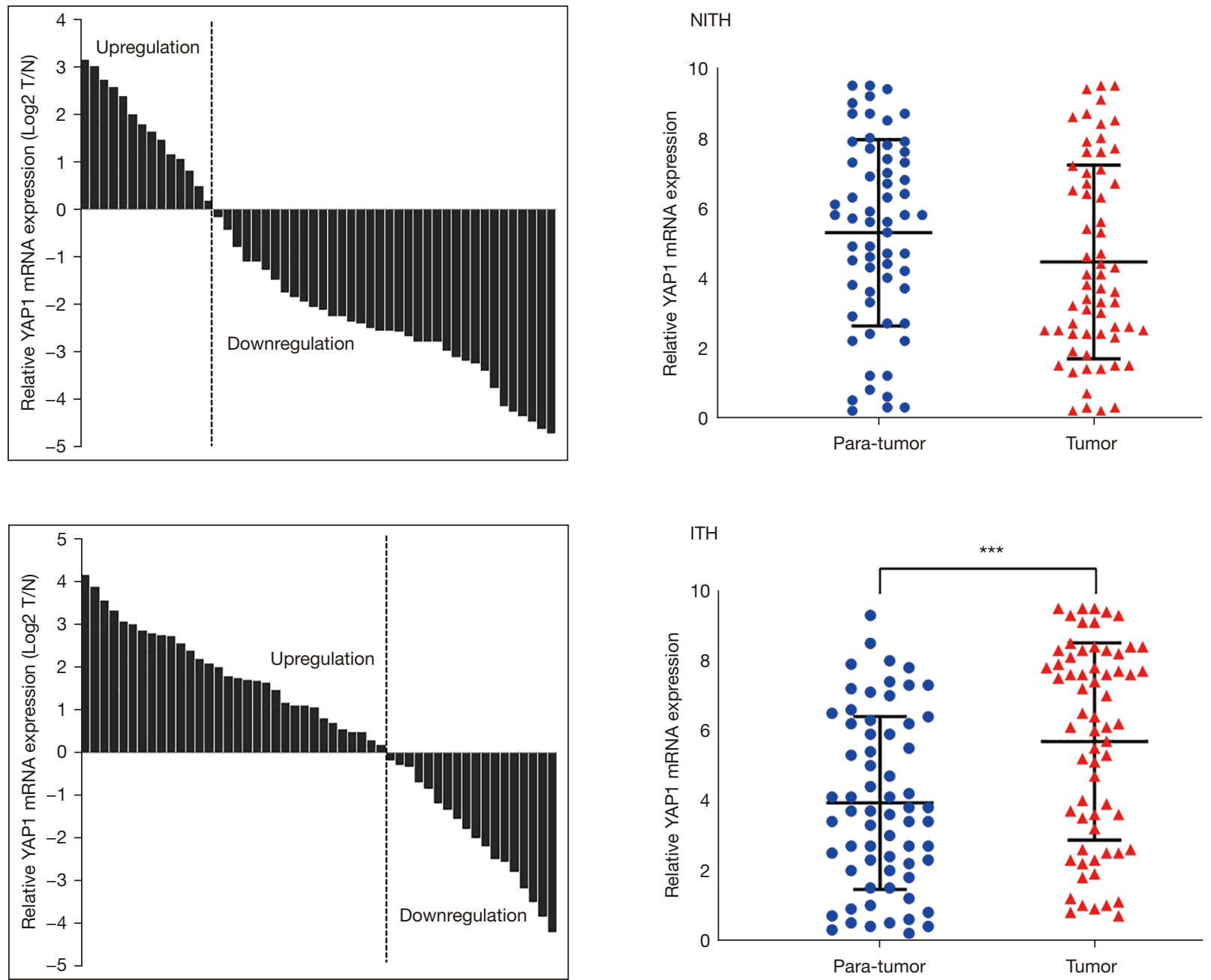

Figure 1 Expression levels of YAP1 messenger ribonucleic acid in 130 paired breast cancer (65 ITH and 65 NITH) and adjacent normal tissues were evaluated by qRT-PCR and statistically analyzed (***, $\mathrm{P}<0.01$ ). YAP1, Yes-associated protein 1; ITH, intratumoral heterogeneity; NITH, non-intratumoral heterogeneity; qRT-PCR, real-time quantitative polymerase chain reaction.

vs. $41.5 \%, \mathrm{P}=0.001)$. To identify the potential difference of YAP1 expression between breast cancer and adjacent normal tissues, we compared the expression levels of YAP1 between paired tumor and para-tumor tissues. As Figure 1 shows, YAP1 expression was higher in ITH patients than NITH patients. A significant difference was also found in the Western blot analysis results for the ITH and NITH tumor tissues (Figure $2 A$ ), which was confirmed by the IHC staining results (Figure 2B). According to the results of the immunofluorescence analysis, co-expression of YAP1 and HER2 was revealed (Figure 2C), and the obvious nuclear localization of YAP1 in ITH patients with recurrent lesions was observed (Figure 2D).
According to the above findings, the overexpression of YAP1 and presence of ITH significantly affected the prognosis of HER2 positive patients. Thus, we further evaluated the continuous and composite measure of recurrence risk, which was determined by a Cox model that included all the above variables. Overall, the breast cancer-free percentage was $75.3 \%(1,242 / 1,650)$, with a range of $95.2 \%$ to $39.1 \%$ among patients with the lowest (0.08) and highest composite risk (5.39), respectively. As Figure 3 shows, the divided point of the composite risk was 2.75 in HER2-ITH patients. When the composite risk was $<2.75$, the survival of the subpopulations was approximate. Thereafter, patients with YAP1 overexpression had a 
A

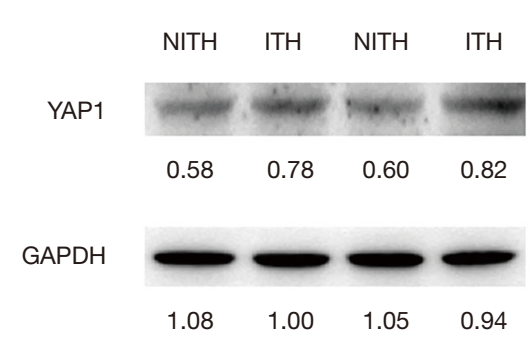

B

C

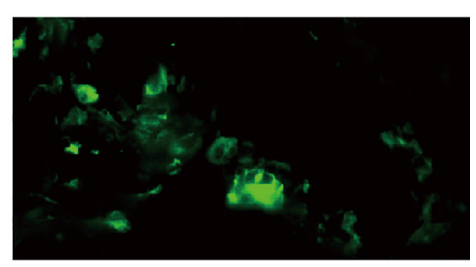

HER2

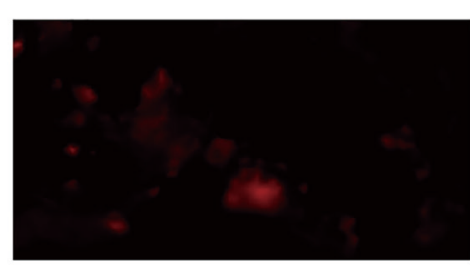

YAP1 + HER2

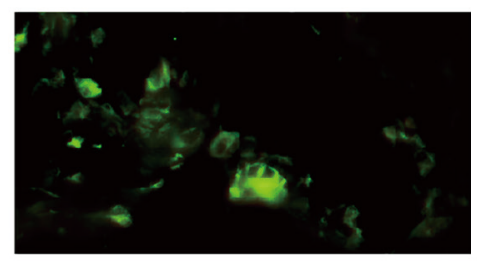

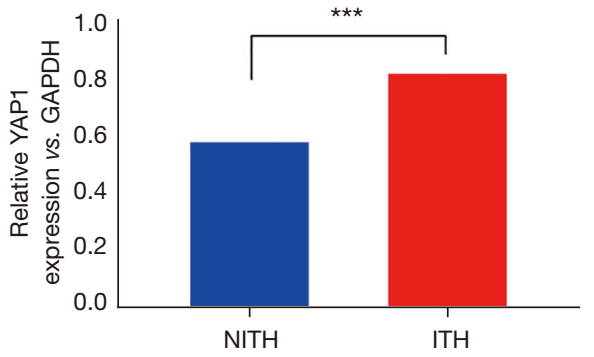

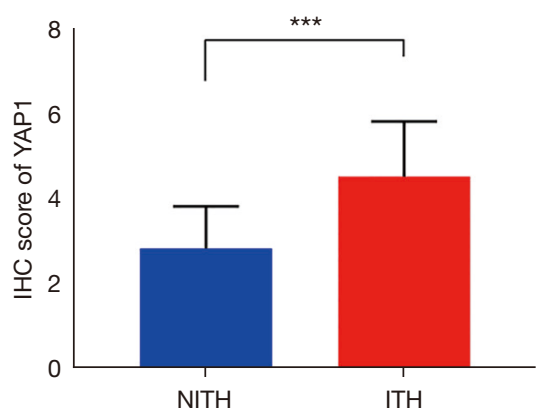

DAPI

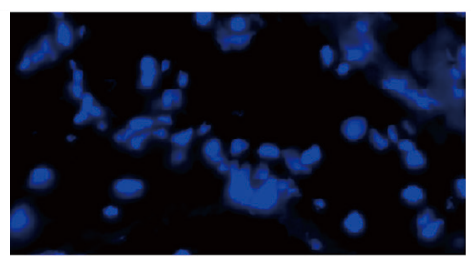

DAPI

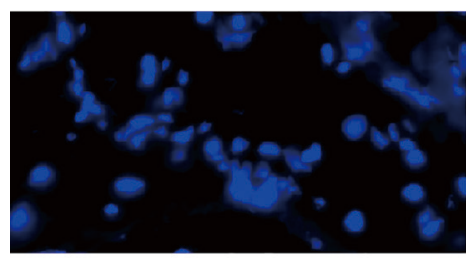

DAPI

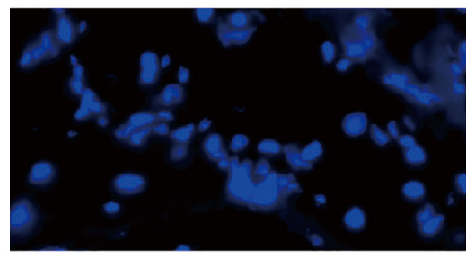

MERGE

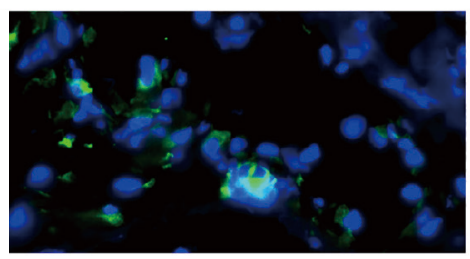

MERCE

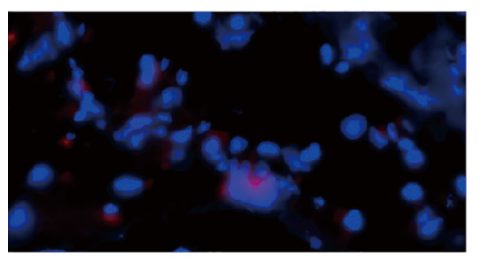

MERGE

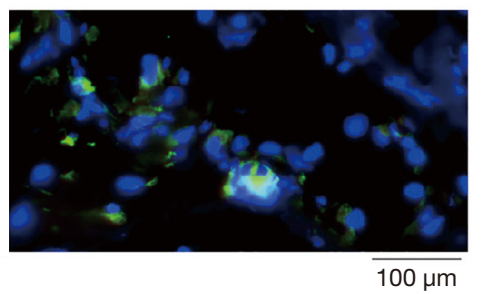


D
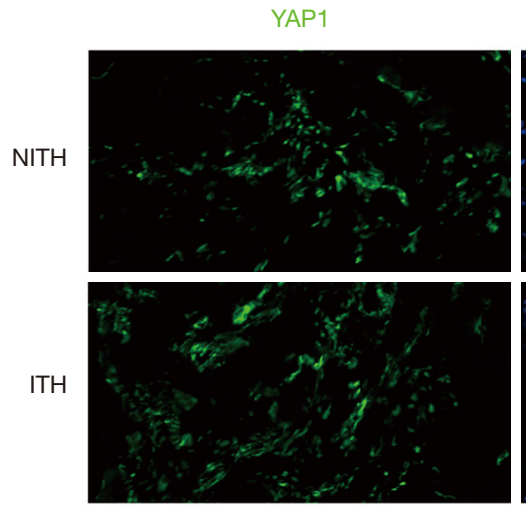

YAP1

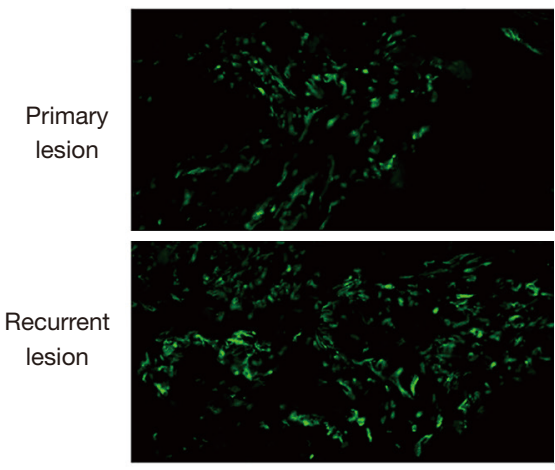

DAPI
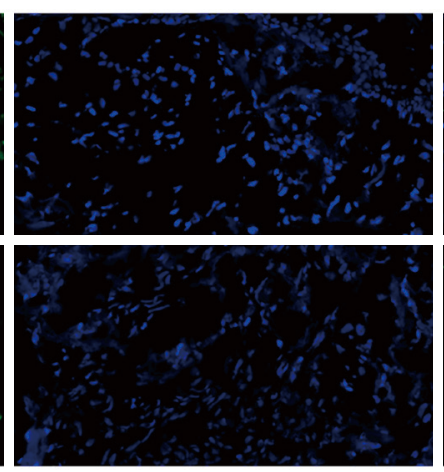

DAPI
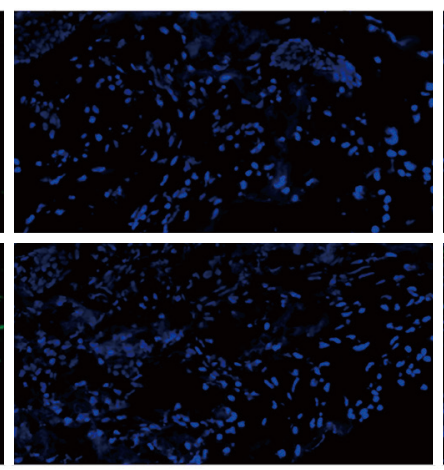
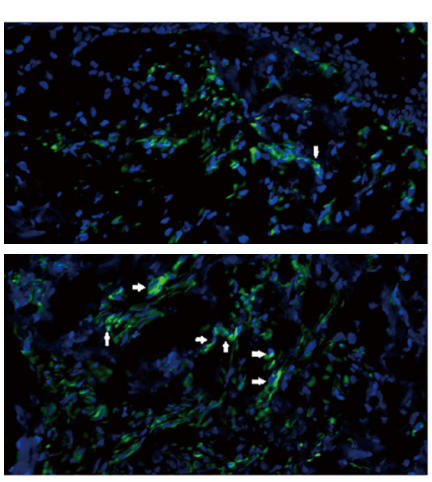

MERGE
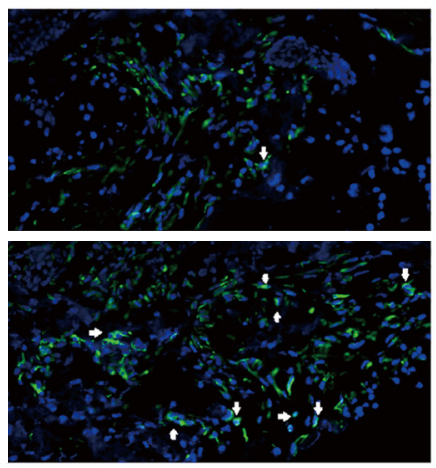

$250 \mu \mathrm{m}$

Figure 2 Representative expression of YAP1 in ITH and NITH tumor tissues as measured by Western blot assays (A) and IHC staining (B). The results of the Western blot assays and IHC staining (scale bar $=250 \mu \mathrm{m}$ ) were both statistically analyzed $(* * *, \mathrm{P}<0.01)$. Co-expression of YAP1 and HER2 (C) (scale bar $=100 \mu \mathrm{m}$ ) as well as comparison of YAP1 nuclear localization in ITH/NITH tissues and primary/ recurrent lesions (D) (scale bar $=250 \mu \mathrm{m}$ ) were both measured by immunofluorescence. YAP1, Yes-associated protein 1; ITH, intratumoral heterogeneity; NITH, non-intratumoral heterogeneity; IHC, immunohistochemistry.

significantly worse prognosis. We observed a tremendous prognostic difference for the highest composite risk (5.39), whereby the proportion of breast cancer-free percentage was $57 \%$ in YAP1-insufficient patients compared to nearly $40 \%$ in YAP1-sufficient patients. Conversely, the divided point of the composite risk according to YAP1 intensity was obviously larger in HER2-NITH cases (3.20) than that in ITH cases (2.80), but survival percentage of NITH cases at the highest composite risk according to YAP1 intensity (50\% vs. $40 \%)$ did not present such a huge difference in comparison with that of ITH cases (56\% vs. $40 \%$ ).

Given the crucial role of ITH in the therapeutic efficacy of Trastuzumab, all of the patients were further divided into 2 groups based on the occurrence of ITH, and their recurrent risk explored. As Figure 4 shows, the prognostically predictive value of YAP1 expression and pLN metastases was more significant in HER2-ITH patients, whereas in NITH patients, the prognostic factors were cTNM stage and LVSI status. Additionally, HER2-NITH patients and YAP1-insufficient patients benefited from a combination of Trastuzumab and Pertuzumab/Lapatinib, while Capecitabine significantly decreased the relapse risk of ITH patients and YAP1-sufficient patients. Similar results were observed in the Kaplan-Meier curve analysis (Figures 5,6).

Based on the above findings, a nomogram analysis was performed to quantify recurrent risk. The risk value was calculated when the pathological and therapeutic information was available (Figure 7). A nomogram was also used to evaluate the median survival time and survival probability of patients (Figure 8). These results provided further evidence of the extent to which YAP1 and ITH affected prognosis. According to the nomogram analysis, 

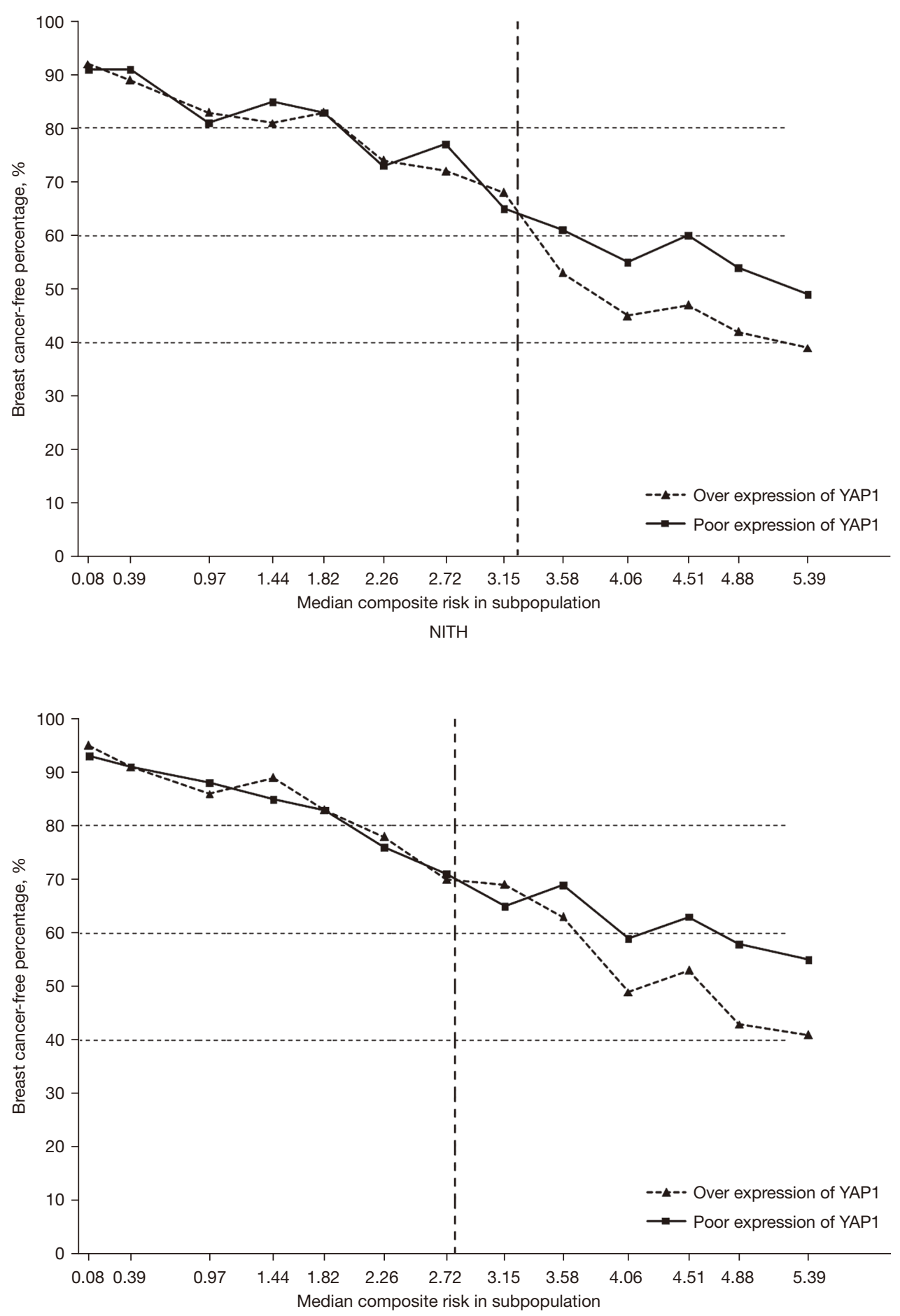

$\mathrm{ITH}$

Figure 3 The survival of the YAP1-related subpopulations were extremely similar when composite risk was lower than the divided point (2.75 for ITH patients and 3.20 for NITH patients). For ITH patients, the prognostic inferiority of the YAP1-sufficient subpopulations was significant, as their breast cancer-free survival was nearly $20 \%$ less than that of the YAP1-insufficient patients when the composite risk reached the highest point of 5.39. Conversely, the breast cancer-free percentage at the highest composite risk did not produce such a huge difference in NITH patients. YAP1, Yes-associated protein 1; ITH, intratumoral heterogeneity; NITH, non-intratumoral heterogeneity. 


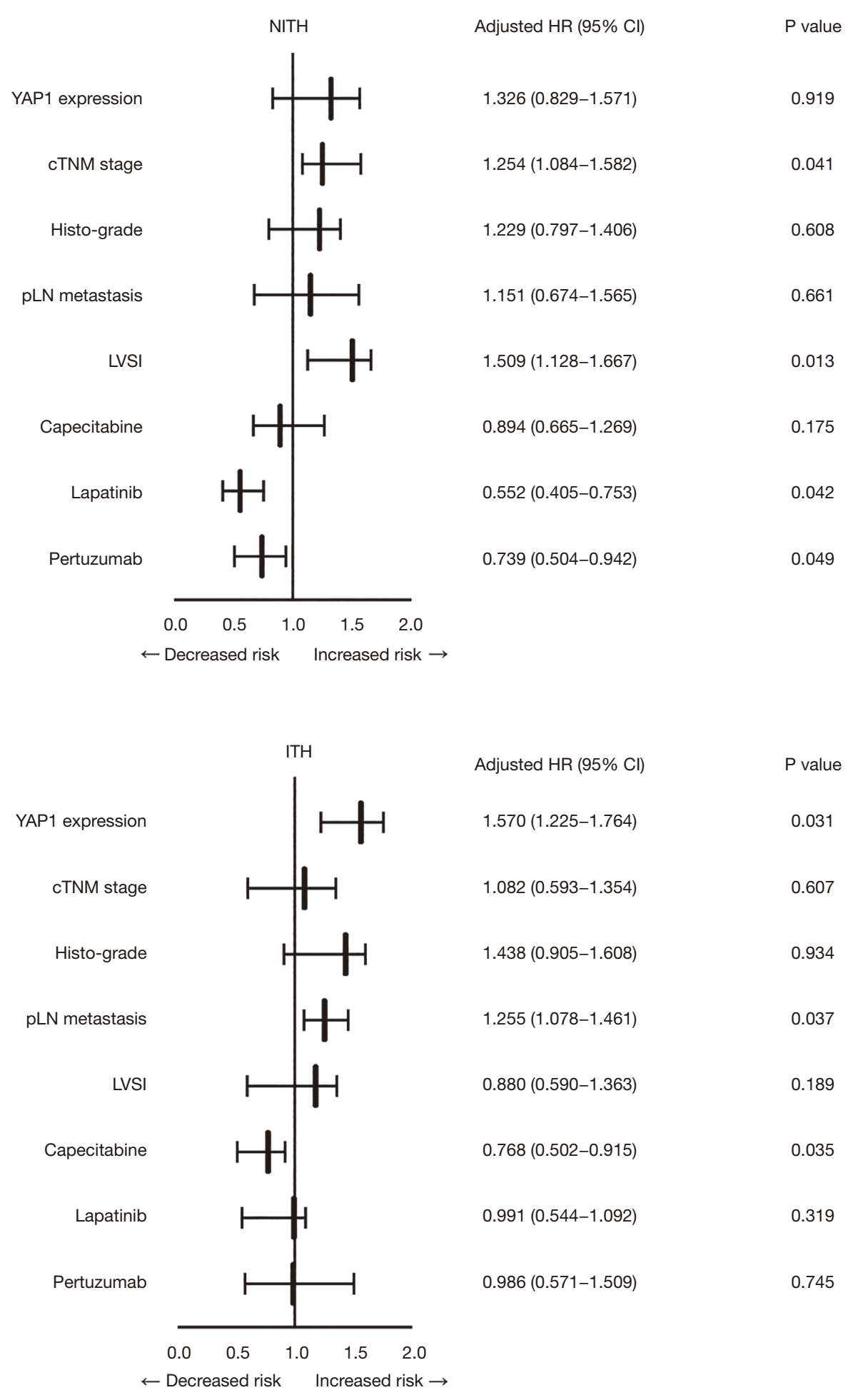

Figure 4 The Cox proportional-hazards model suggested that YAP1 overexpression and pLN metastases increased the recurrent risk of HER2-ITH patients. Similarly, advanced cTNM stage and LVSI status increased the recurrent risk of NITH patients. The combination of Trastuzumab and Pertuzumab/Lapatinib significantly decreased the relapse risk of NITH patients, while Capecitabine significantly decreased the relapse risk of ITH patients. YAP1, Yes-associated protein 1; HER2, human epidermal growth factor receptor 2; ITH, intratumoral heterogeneity; LVSI, lymph-vascular space invasion; NITH, non-intratumoral heterogeneity. 

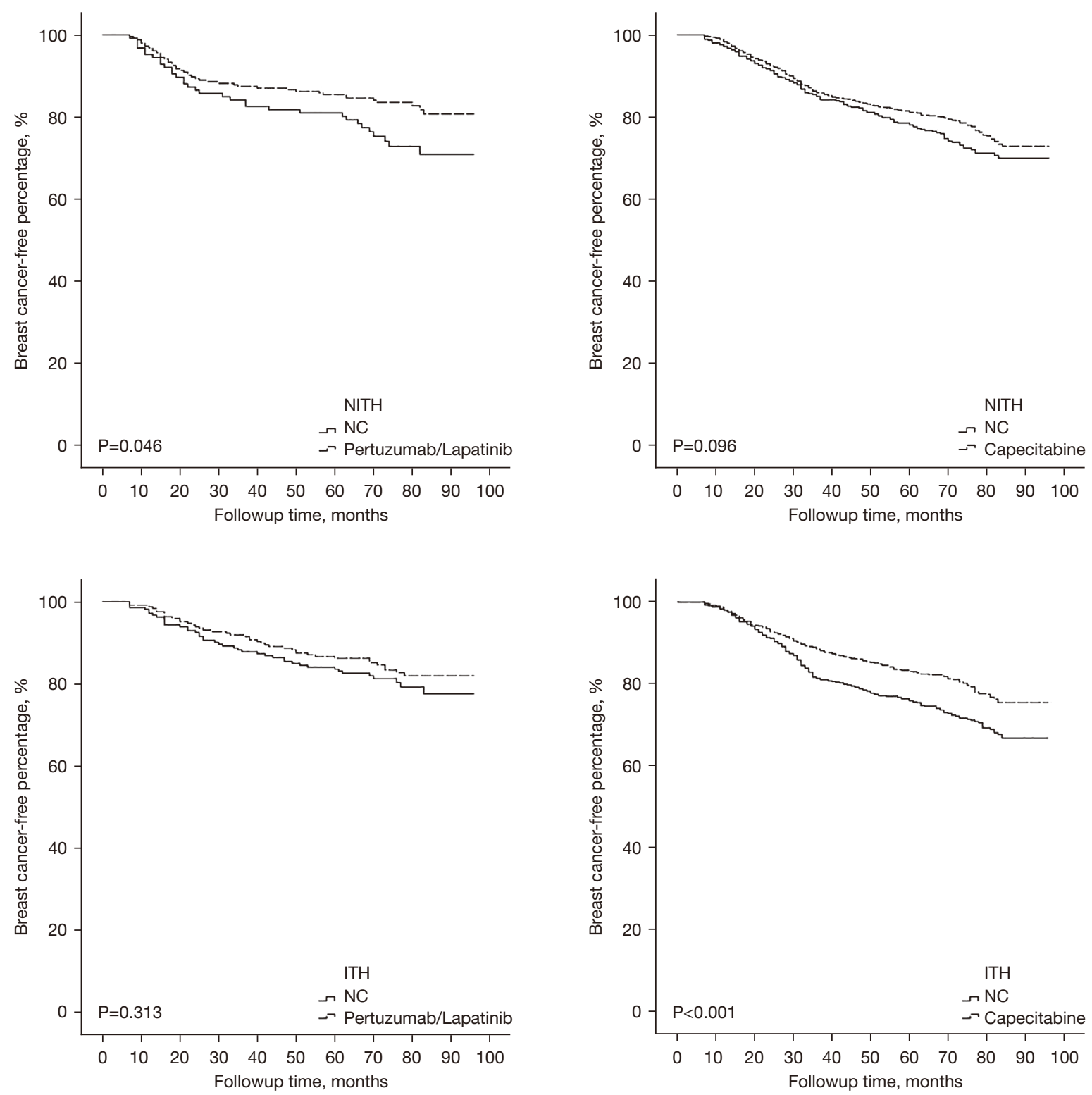

Figure 5 The Kaplan-Meier survival analysis showed that the intensified treatment of Pertuzumab/Lapatinib significantly improved the survival of HER2-NITH patients, while the intensified treatment of Capecitabine significantly improved the survival of HER2-ITH patients. HER2, human epidermal growth factor receptor 2; ITH, intratumoral heterogeneity; NITH, non-intratumoral heterogeneity.

we visually assessed the potential of individual intensified regimes for subpopulations.

\section{Discussion}

Trastuzumab has been shown to greatly improve the survival of and significantly reduce the relapse risk of HRE2 positive breast cancer patients (19-21). Trastuzumab-based adjuvant systemic therapy has also been found to improve therapeutic efficacy by generally prolonging cancer-free survival (21). Despite these successes, it should be noted that some HER2 positive patients did not display the expected outcome, which might be due to the existence of HER2 heterogeneity. However, to date, the prognostic effect of HER2 heterogeneity has not been well established by research. 

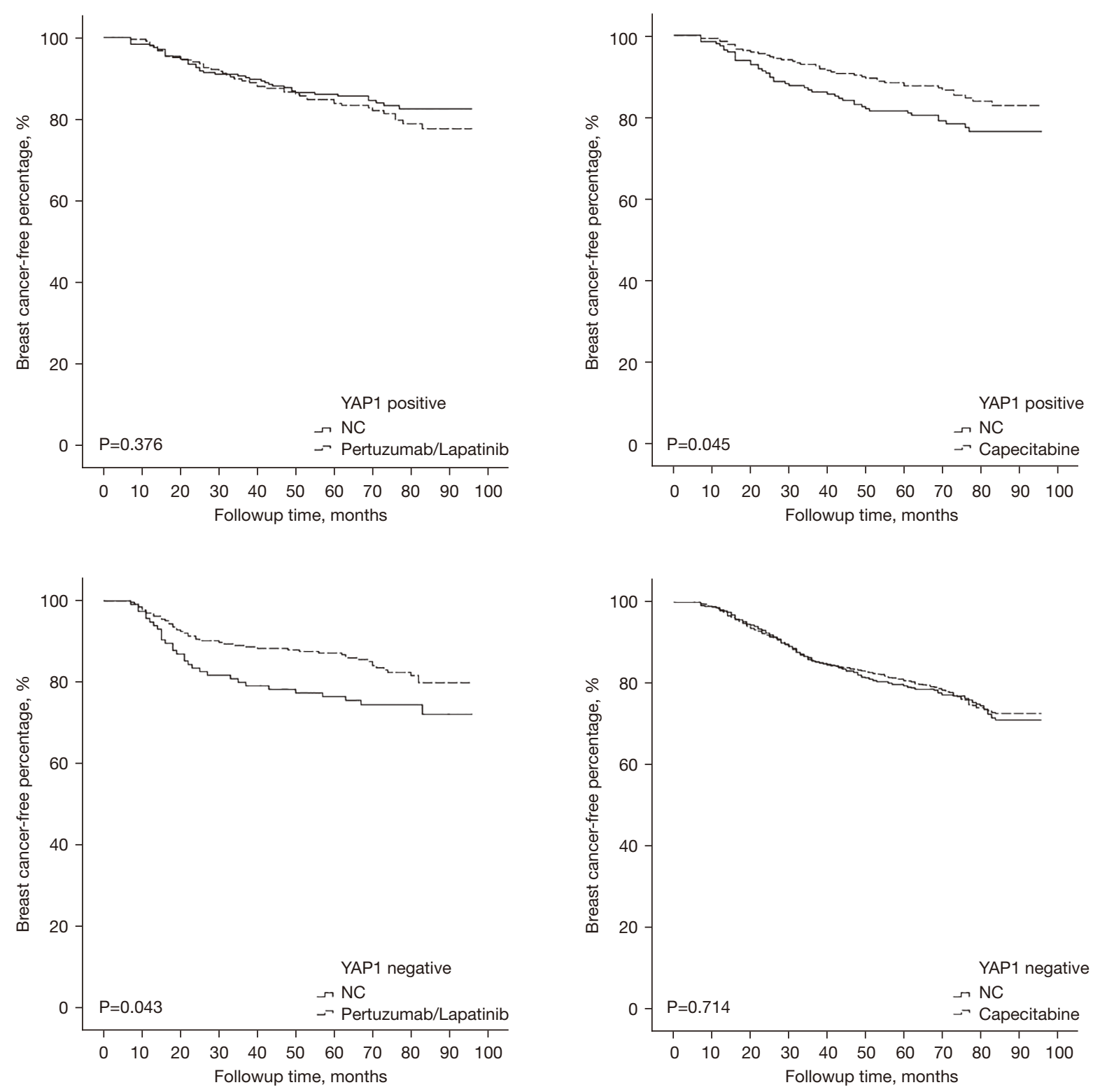

Figure 6 The Kaplan-Meier survival analysis showed that the intensified treatment of Pertuzumab/Lapatinib significantly improved the survival of YAP1 insufficient patients, while the intensified treatment of Capecitabine significantly improved the survival of YAP1 overexpression patients. YAP1, Yes-associated protein 1.

Previous studies have found a difference in HER2 intensity between primary and recurrent lesions. Notably, Burstein et al. reported that among patients who received a normative treatment of paclitaxel and Trastuzumab, $26.1 \%$ of recurrent lesions showed a decrease in HER2 amplification compared to primary lesions, which suggests that a decline in HER2 intensity diminishes the therapeutic efficacy of Trastuzumab and increases the relapse risk (22).
Harris et al. and Perez et al. reported that up to $12 \%$ of primary HER2 positive cases showed decreased HER2 expression in their recurrent lesions, which impaired their survival outcomes $(23,24)$. Hurley et al. showed that $43 \%$ of patients with recurrent lesions who were initially HER2 FISH positive became FISH negative, which might reduce the prognostic benefit associated with treatments of Trastuzumab, docetaxel, and cisplatin (25). In this study, we 


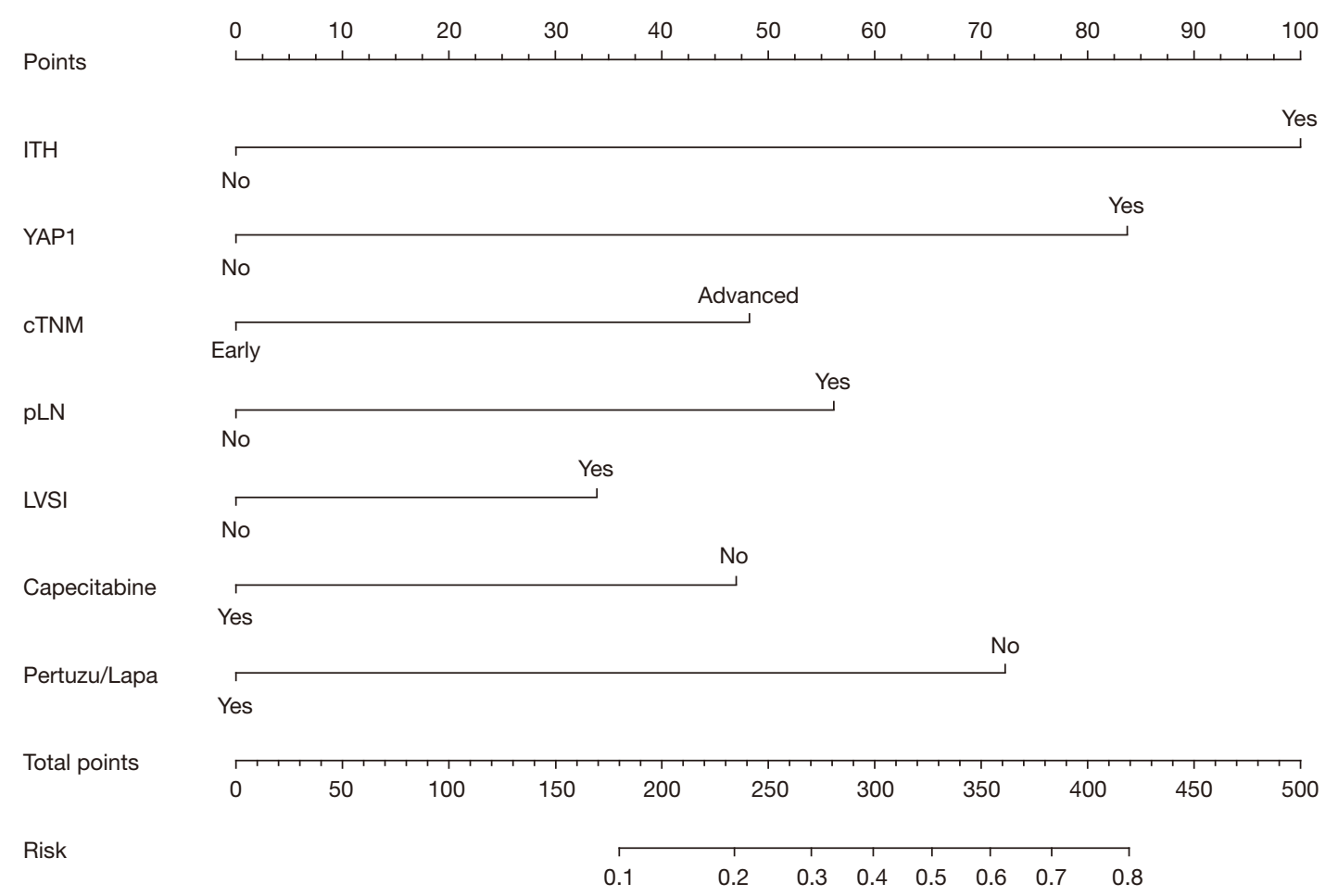

Figure 7 Nomogram for recurrent risk estimation of HR-/HER2+ breast cancer patients. The value of each variable was given a score on the point scale axis in the first line. To use the nomogram, a vertical line was drawn to the point scale axis to determine the number of points attributable to each variable value. A total score was easily calculated by adding each single score. The sum was then located on the total points axis, and the total score was projected to the bottom line of the nomogram to estimate the probability of recurrent risk. HR, hormone receptor; HER2, human epidermal growth factor receptor 2; LVSI, lymph-vascular space invasion.

found that patients with HER2 heterogeneity also tended to have insufficient HER2 expression and amplification in recurrent lesions. Notably, this subpopulation generally suffered from poor outcomes even if they underwent normative Trastuzumab-based systemic therapy.

Previous pre-clinical research had shown that the overexpression of YAP1 is significantly associated with the migration and invasion of breast cancer cells, and induces the inhibition of apoptosis and anchorage-independent growth, which contributes to tumorigenic transformation $(11,12)$. The knockdown of YAP1 has been shown to reduce migration and invasion, and suppress tumor development and metastasis in a breast cancer mouse model $(26,27)$. Additionally, the depletion of YAP1 was significant in basal and epithelial cells, and promoted luminal differentiation and acted as a luminal to basal phenotype trigger (28). Conversely, YAP1 has been shown to be correlated with tumor metastases and drug resistance, which obviously affect patients' survival. Given their crucial effect on the outcome of HER2 positive patients, we evaluated the relationship between YAP1 expression and HER2-ITH in this study. Notably, we observed both an overexpression of YAP1 and insufficient HER2 intensity in HER2-ITH patients. Patients with YAP1 overexpression suffered from poor prognostic outcomes, especially when the HER2 signal intensity was insufficient. These findings suggest that the upregulation of YAP1 expression leads to poor outcomes for HER2-ITH patients.

Technologic advancements have made it possible to measure variables on a continuous basis. Cancer-related clinical studies have quantitatively assessed selected variables, evaluated their clinical outcome and prognostic effectiveness, and identified the subgroups that are most likely to benefit from a particular treatment modality. However, the associations between risk factors and prognostic outcomes might not follow a linear pattern, and cannot be detected with simple methods. Our study found that the prognostic outcomes of HER2-ITH patients 


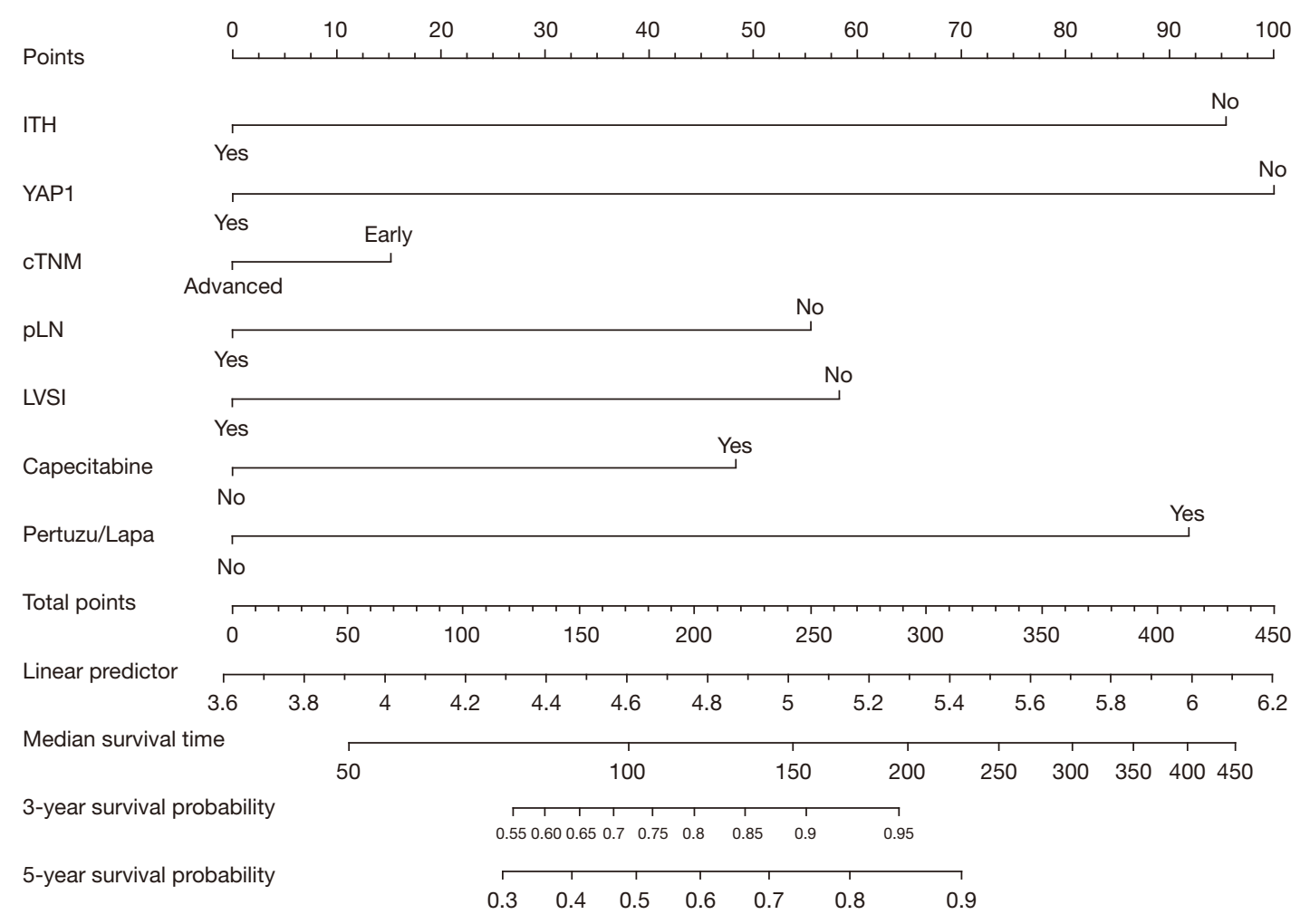

Figure 8 Nomogram for survival probability estimation of HR-/HER2 + breast cancer patients. Each predictive factor in the Cox proportional-hazards model was given a score in the gram. The final score was the sum of all single scores, which was then projected to the bottom line of the gram to predict the median survival time and 3-year/5-year survival probability. HR, hormone receptor; HER2, human epidermal growth factor receptor 2; LVSI, lymph-vascular space invasion.

depended on distinct factors, and that any evaluation of relapse risk should also consider the interactions of these factors. However, improved analysis techniques are needed to estimate these complex connections (6).

We found that the STEPP methodology was useful for evaluating prognostic heterogeneity, especially when a certain risk factor was obviously correlated with a particular prognostic feature. Prognostic heterogeneity was illustrated graphically according to competing risks, which enabled us to conduct a convenient exploratory evaluation. Statistical approaches, such as STEPP, enabled us to identify potential predictive factors of recurrent risk candidates.

In this study, the STEPP methodology was used to evaluate the prognosis of breast cancer patients with or without HER2 heterogeneity, and determine the prognostic effectiveness of HER2-ITH according to the composite risk in subpopulations. We summarized the breast cancerfree percentage of ITH and NITH patients, and found that HER2 heterogeneity was associated with a poor breast cancer-free interval. Further, we noted that subpopulations with a high composite risk had the greatest magnitude of YAP1 expression. As the composite risk increased, the prognostic inferiority of YAP1 overexpression patients became more and more obvious. Further, we found that the combination of HER2-ITH and YAP1 overexpression tended to lead to a poor outcome, even if the patients underwent normative systemic therapy.

We also examined whether this subpopulation would benefit from intensive adjuvant therapy. According to our findings, HER2-NITH patients benefit from the combination of Trastuzumab and Pertuzumab/Lapatinib. The combination also improved the survival of YAP1insufficient patients. Conversely, patients with HER2ITH and/or YAP1 overexpression tended to benefit from Capecitabine. These results demonstrated that the heterogeneity of HER2 and YAP1 generally signified the individuation of treatment, especially in patients with a high recurrent risk. Based on the identification of HER2-ITH 
and YAP1 overexpression, the above intensified treatment should be performed in specific populations as early as possible.

As a single-center clinical retrospective study, we note the limitations of this work. Multi-center prospective studies need to be conducted to confirm our conclusions in the future. As a defect of STEPP, the selection of an end point would potentially affect the interpretation of results and the effect of the results on clinical practice. Statistical methods need to be continuously improved in clinical studies. Further analyses and the updating of our findings according to the ongoing follow-up results are also needed.

\section{Conclusions}

In conclusion, YAP1 overexpression and nuclear localization usually occurred in HER2-ITH patients. For HER2NITH patients, advanced cTNM stage and LVSI status increased recurrent risk, and intensified Pertuzumab or Lapatinib treatment (in combination with Trastuzumab) improved patient survival. For HER2-ITH patients, YAP1 overexpression and $\mathrm{pLN}$ metastases increased recurrent risk, and intensified Capecitabine treatment improved patient survival. YAP1 overexpression contributed to a poor prognostic outcome, especially when HER2 signal intensity was insufficient.

\section{Acknowledgments}

The authors are grateful to Dr. Xue Dong and Dr. Xin Li for proofreading this manuscript.

Funding: Our study was supported by the Clinical Research Fund of the Wu Jie-Ping Medical Foundation (No. 320.6750.2020-20-28), Project of Hunan Provincial Health Committee (No. 202204082512), and the Launching Fund of the Xiangya Hospital Fuqing Postdoctoral Program (No. 2209090555211).

\section{Footnote}

Reporting Checklist: The authors have completed the MDAR checklist. Available at https://gs.amegroups.com/article/ view/10.21037/gs-22-52/rc

Data Sharing Statement: Available at https://gs.amegroups. com/article/view/10.21037/gs-22-52/dss

Conflicts of Interest: All authors have completed the ICMJE uniform disclosure form (available at https://gs.amegroups. com/article/view/10.21037/gs-22-52/coif). The authors have no conflicts of interest to declare.

Ethical Statement: The authors are accountable for all aspects of the work in ensuring that questions related to the accuracy or integrity of any part of the work are appropriately investigated and resolved. The study was conducted in accordance with the Declaration of Helsinki (as revised in 2013). The study was approved by the Xiangya Hospital Ethics Committee (approval No. 202004189) and individual consent for this retrospective analysis was waived.

Open Access Statement: This is an Open Access article distributed in accordance with the Creative Commons Attribution-NonCommercial-NoDerivs 4.0 International License (CC BY-NC-ND 4.0), which permits the noncommercial replication and distribution of the article with the strict proviso that no changes or edits are made and the original work is properly cited (including links to both the formal publication through the relevant DOI and the license). See: https://creativecommons.org/licenses/by-nc-nd/4.0/.

\section{References}

1. Mittendorf EA, Wu Y, Scaltriti M, et al. Loss of HER2 amplification following trastuzumab-based neoadjuvant systemic therapy and survival outcomes. Clin Cancer Res 2009;15:7381-8.

2. Hudis CA. Trastuzumab--mechanism of action and use in clinical practice. N Engl J Med 2007;357:39-51.

3. Perez EA, Romond EH, Suman VJ, et al. Four-year follow-up of trastuzumab plus adjuvant chemotherapy for operable human epidermal growth factor receptor 2-positive breast cancer: joint analysis of data from NCCTG N9831 and NSABP B-31. J Clin Oncol 2011;29:3366-73.

4. Gianni L, Eiermann W, Semiglazov V, et al. Neoadjuvant chemotherapy with trastuzumab followed by adjuvant trastuzumab versus neoadjuvant chemotherapy alone, in patients with HER2-positive locally advanced breast cancer (the NOAH trial): a randomised controlled superiority trial with a parallel HER2-negative cohort. Lancet 2010;375:377-84.

5. Pectasides D, Gaglia A, Arapantoni-Dadioti P, et al. HER$2 /$ neu status of primary breast cancer and corresponding metastatic sites in patients with advanced breast cancer treated with trastuzumab-based therapy. Anticancer Res 
2006;26:647-53.

6. Wolff AC, Hammond ME, Hicks DG, et al. Recommendations for human epidermal growth factor receptor 2 testing in breast cancer: American Society of Clinical Oncology/College of American Pathologists clinical practice guideline update. J Clin Oncol 2013;31:3997-4013.

7. Royston P, Altman DG, Sauerbrei W. Dichotomizing continuous predictors in multiple regression: a bad idea. Stat Med 2006;25:127-41.

8. Bartucci M, Dattilo R, Moriconi C, et al. TAZ is required for metastatic activity and chemoresistance of breast cancer stem cells. Oncogene 2015;34:681-90.

9. Mittendorf EA, Storrer CE, Shriver CD, et al. Investigating the combination of trastuzumab and HER2/ neu peptide vaccines for the treatment of breast cancer. Ann Surg Oncol 2006;13:1085-98.

10. Zhao B, Wei X, Li W, et al. Inactivation of YAP oncoprotein by the Hippo pathway is involved in cell contact inhibition and tissue growth control. Genes Dev 2007;21:2747-61.

11. Mi W, Lin Q, Childress C, et al. Geranylgeranylation signals to the Hippo pathway for breast cancer cell proliferation and migration. Oncogene 2015;34:3095-106.

12. Chan SW, Lim CJ, Guo K, et al. A role for TAZ in migration, invasion, and tumorigenesis of breast cancer cells. Cancer Res 2008;68:2592-8.

13. Bonetti M, Zahrieh D, Cole BF, et al. A small sample study of the STEPP approach to assessing treatment-covariate interactions in survival data. Stat Med 2009;28:1255-68.

14. Peto R, Pike MC, Armitage P, et al. Design and analysis of randomized clinical trials requiring prolonged observation of each patient. II. analysis and examples. Br J Cancer 1977;35:1-39.

15. Dignam JJ, Kocherginsky MN. Choice and interpretation of statistical tests used when competing risks are present. J Clin Oncol 2008;26:4027-34.

16. Hudis CA, Barlow WE, Costantino JP, et al. Proposal for standardized definitions for efficacy end points in adjuvant breast cancer trials: the STEEP system. J Clin Oncol 2007;25:2127-32.

17. Francis PA, Regan MM, Fleming GF, et al. Adjuvant ovarian suppression in premenopausal breast cancer. $\mathrm{N}$ Engl J Med 2015;372:436-46.

18. Pagani O, Regan MM, Walley BA, et al. Adjuvant exemestane with ovarian suppression in premenopausal breast cancer. N Engl J Med 2014;371:107-18.
19. Romond EH, Perez EA, Bryant J, et al. Trastuzumab plus adjuvant chemotherapy for operable HER2-positive breast cancer. N Engl J Med 2005;353:1673-84.

20. Viani GA, Afonso SL, Stefano EJ, et al. Adjuvant trastuzumab in the treatment of her-2-positive early breast cancer: a meta-analysis of published randomized trials. BMC Cancer 2007;7:153.

21. Mendes D, Alves C, Afonso N, et al. The benefit of HER2-targeted therapies on overall survival of patients with metastatic HER2-positive breast cancer--a systematic review. Breast Cancer Res 2015;17:140.

22. Burstein HJ, Harris LN, Gelman R, et al. Preoperative therapy with trastuzumab and paclitaxel followed by sequential adjuvant doxorubicin/cyclophosphamide for HER2 overexpressing stage II or III breast cancer: a pilot study. J Clin Oncol 2003;21:46-53.

23. Harris LN, You F, Schnitt SJ, et al. Predictors of resistance to preoperative trastuzumab and vinorelbine for HER2-positive early breast cancer. Clin Cancer Res 2007;13:1198-207.

24. Perez EA, Suman VJ, Davidson NE, et al. HER2 testing by local, central, and reference laboratories in specimens from the North Central Cancer Treatment Group N9831 intergroup adjuvant trial. J Clin Oncol 2006;24:3032-8.

25. Hurley J, Doliny P, Reis I, et al. Docetaxel, cisplatin, and trastuzumab as primary systemic therapy for human epidermal growth factor receptor 2-positive locally advanced breast cancer. J Clin Oncol 2006;24:1831-8.

26. Skibinski A, Breindel JL, Prat A, et al. The Hippo transducer TAZ interacts with the SWI/SNF complex to regulate breast epithelial lineage commitment. Cell Rep 2014;6:1059-72.

27. Overholtzer M, Zhang J, Smolen GA, et al. Transforming properties of YAP, a candidate oncogene on the chromosome 11q22 amplicon. Proc Natl Acad Sci U S A 2006;103:12405-10.

28. Chen Q, Zhang N, Gray RS, et al. A temporal requirement for Hippo signaling in mammary gland differentiation, growth, and tumorigenesis. Genes Dev 2014;28:432-7.

(English Language Editor: L. Huleatt)

Cite this article as: Yuan JQ, Xiao Z, Wang SM, Guo L. The prognostic effect of HER2 heterogeneity and YAP1 expression in HER2 positive breast cancer patients: a retrospective study. Gland Surg 2022;11(2):451-465. doi: 10.21037/gs-22-52 